\title{
HIGHER ORDER ASYMPTOTIC BEHAVIOR OF CERTAIN KÄHLER METRICS AND UNIFORMIZATION FOR STRONGLY PSEUDOCONVEX DOMAINS
}

\author{
Jae-Cheon Joo and Aeryeong Seo
}

\begin{abstract}
We provide some relations between CR invariants of boundaries of strongly pseudoconvex domains and higher order asymptotic behavior of certain complete Kähler metrics of given domains. As a consequence, we prove a rigidity theorem of strongly pseudoconvex domains by asymptotic curvature behavior of metrics.
\end{abstract}

\section{Introduction}

The characterization of holomorphic covering spaces of high dimensional complex manifolds is complicated as against the Riemann uniformization theorem for Riemann surfaces. In differential geometric point of view, it is believed that the curvature behaviors of certain Kähler metrics would restrict the complex structure of manifolds. For instance, Siu-Yau have proved in [17] the Frankel conjecture that a compact Kähler manifold with positive bisectional curvature is biholomorphic to the complex projective space. See also [10] for its generalization. They also have proved that a simply connected complete Kähler manifold is biholomorphic to the complex Euclidean space if the sectional curvature is nonpositive and asymptotically vanishes faster than quadratic order. See [16]. Siu also proved in [15] that the complex structure of a compact Kähler manifold with strongly negative curvature tensor is determined by its homotopy type. This is called Siu's strong rigidity theorem.

The aim of this paper is to find a curvature condition of some noncompact manifolds which are covered by the unit ball. As a generalization of Chern-Ji's theorem $([2])$, Nemirovskii-Shaffikov proved in $[11,12]$ that a strongly pseudoconvex domain $\Omega$ with $C^{\infty}$-smooth boundary is covered by the unit ball if every boundary point is spherical in the sense that all the CR invariants vanish

Received March 25, 2014.

2010 Mathematics Subject Classification. 32V15, 53B35.

Key words and phrases. Bergman metric, Graham-Lee connection, CR invariants.

The second author was supported by National Researcher Program of the National Research Foundation (NRF) funded by the Ministry of Science, ICT \& Future Planning (No.2014028806). 
identically on $\partial \Omega$ (cf. [3]). Therefore, it is needed to find relations between boundary $\mathrm{CR}$ invariants and curvature behavior of interior metrics if we want to characterize the curvature condition under that a strongly pseudoconvex domain is covered by the unit ball. Let $\Omega$ be a bounded strongly pseudoconvex domain in $\mathbb{C}^{n+1}$ with $C^{\infty}$-smooth boundary. Suppose $\varphi$ is a $C^{k}$-smooth defining function of $\Omega$ for some $k \geq 4$ and $-\log (-\varphi)$ is strongly plurisubharmonic so that

$$
g_{i \bar{j}}=-\frac{\partial^{2}}{\partial z_{i} \partial z_{\bar{j}}} \log (-\varphi)
$$

defines a complete Kähler metric on $\Omega$. For instance, if $\varphi(z)=-K(z, z)^{-\frac{1}{n+1}}$ where $K$ is the Bergman kernel on $\Omega,\left(g_{i \bar{j}}\right)$ is a constant multiple of the Bergman metric and if $\varphi$ is the solution of the complex Monge-Ampére equation

$$
\begin{cases}J(\varphi)=1, & \text { on } \Omega \\ \varphi \equiv 0, & \text { on } \partial \Omega\end{cases}
$$

where

$$
J(\varphi)=(-1)^{n}\left(\begin{array}{cc}
\varphi & \frac{\partial \varphi}{\partial z_{\bar{j}}} \\
\frac{\partial \varphi}{\partial z_{k}} & \frac{\partial^{2} \varphi}{\partial z_{k} \partial z_{\bar{j}}}
\end{array}\right),
$$

$\left(g_{i \bar{j}}\right)$ is the Kähler-Einstein metric on $\Omega$.

In 1978, Klembeck ([8]) proved that the holomorphic sectional curvature of the Bergman metric tends to a negative constant as the point of evaluation tending to the boundary by means of the asymptotic expansion formula for the Bergman kernel given by Fefferman ([4]). Klembeck's theorem bas been generalized by Kim and $\mathrm{Yu}$ in $C^{2}$-smooth boundary case $([7])$ and also reproved by Barletta in [1]. The main theorem of this paper is as follows.

Theorem 1.1. Let $\Omega$ be a bounded strongly pseudoconvex domain in $\mathbb{C}^{n+1}$ with $C^{\infty}$-smooth boundary and $\varphi$ be a $C^{k}$-smooth defining function of $\Omega$ for some $k \geq 4$ such that

$$
g_{i \bar{j}}=-\frac{\partial^{2}}{\partial z_{i} \partial z_{\bar{j}}} \log (-\varphi)
$$

is a complete Kähler metric on $\Omega$. For $p \in \Omega, W \in \mathbb{C}^{n+1} /\{0\}$, denote $H_{p}(W)$ the holomorphic sectional curvature of $\left(g_{i \bar{j}}\right)$ at $p$ in direction of $W$. Then

(1) For $n \geq 2$, if

$$
H_{p}(W)=-2+O\left(\varphi^{2}\right)
$$

then $\partial \Omega$ is spherical at every point.

(2) For $n=1$, if $k \geq 5$ and if

$$
H_{p}(W)=-2+O\left(\varphi^{3}\right),
$$

then $\partial \Omega$ is spherical at every point. 
By the Nemirovskii-Shaffikov theorem, the domain $\Omega$ is holomorphically covered by the unit ball if either (2) or (3) in Theorem 1.1 is satisfied. In this theorem, we do not assume the $C^{\infty}$-smoothness of the defining function $\varphi$, since the Bergman metric and the Kähler-Einstein metric that are most important two examples, are not defined by $C^{\infty}$-smooth functions even though the boundary is $C^{\infty}$-smooth.

Throughout this paper, the Greek indices $\alpha, \beta, \gamma, \ldots$ run from 1 to $n$ and the Roman indices $i, j, k, \ldots$ run from 0 to $n$. Denote by $O\left(\varphi^{k}\right)$ a function that $\frac{O\left(\varphi^{k}\right)}{\varphi^{k}}$ is bounded as $\varphi \rightarrow 0$. We also make use of the summation convention.

\section{The Graham-Lee connection}

Let $\Omega$ be a strongly pseudoconvex domain in $\mathbb{C}^{n+1}$ with $C^{\infty}$-smooth boundary. Let $\varphi$ be a $C^{k}$-smooth defining function of $\Omega=\left\{z \in \mathbb{C}^{n+1}: \varphi(z)<0\right\}$ for some $k \geq 4$. Let $U=\{z \in \Omega:-\widetilde{\varepsilon}<\varphi \leq 0\}$ for sufficiently small $\widetilde{\varepsilon}>0$ and $M^{\varepsilon}=\{\varphi=-\varepsilon\}$ for $\varepsilon$ satisfying $\widetilde{\varepsilon}>\varepsilon \geq 0$. Let $\mathcal{H}$ be an complex vector bundle on $U$ with $\mathcal{H}_{p}=T^{1,0} \mathbb{C}^{n+1} \cap \mathbb{C} T_{p} M^{\varepsilon}$ for $p \in M^{\varepsilon}$. Let $\theta=\operatorname{Im} \partial \varphi$. Then the restriction of $\theta$ on $M^{\varepsilon}$ defines a pseudohermitian structure on $M^{\varepsilon}$. Let $T$ be the characteristic vector field for $\left(M^{\varepsilon}, \theta\right)$. That is, $T$ is the tangent vector for $\left(M^{\varepsilon}, \theta\right)$ satisfying $\theta(T)=1$ and $\left.T\right\lrcorner\left. d \theta\right|_{\mathcal{H}}=0$. Let $N=-J T$ where $J$ is the standard almost complex structure on $\mathbb{C}^{n}$ and

$$
\xi=\frac{1}{2}(N-i T)
$$

Note that $\partial \varphi(\xi)=1$ and $d \theta=i \partial \bar{\partial} \varphi$. Let $\left\{W_{\alpha}\right\}$ be a local frame of $\mathcal{H}$. Let $W_{0}=\xi$ and $\theta^{0}=\partial \varphi$. Then $\left\{W_{0}, W_{\alpha}\right\}$ is a local frame for $T^{1,0} U$. Let $\left\{\theta^{0}, \theta^{\alpha}\right\}$ be its coframe. Then there exists a positive definite hermitian symmetric matrix $\left(h_{\alpha \bar{\beta}}\right)$ such that

$$
d \theta^{0}=-\partial \bar{\partial} \varphi=-h_{\alpha \bar{\beta}} \theta^{\alpha} \wedge \theta^{\bar{\beta}}-r \theta^{0} \wedge \theta^{\overline{0}}
$$

where $r=2 \partial \bar{\partial} \varphi(\xi, \bar{\xi})$. Since $d \varphi=0$ for each $M^{\varepsilon}$, we have on $M^{\varepsilon}$

$$
d \theta=i h_{\alpha \bar{\beta}} \theta^{\alpha} \wedge \theta^{\bar{\beta}} \text {. }
$$

Then by the pseudohermitian structure in [18], there exist 1-forms $\left(\omega_{\beta}{ }^{\alpha}\right)$ and $\tau^{\alpha}=A^{\alpha}{ }_{\bar{\beta}} \theta^{\bar{\beta}}$ on $M^{\varepsilon}$ such that

$$
\begin{gathered}
d \theta^{\alpha}=\theta^{\beta} \wedge \omega_{\beta}^{\alpha}+\theta \wedge \tau^{\alpha}, \\
d h_{\alpha \bar{\beta}}=\omega_{\alpha \bar{\beta}}+\omega_{\bar{\beta} \alpha}, \text { and } A_{\alpha \beta}=A_{\beta \alpha},
\end{gathered}
$$

where $\omega_{\alpha \bar{\beta}}=\omega_{\alpha}^{\gamma} h_{\gamma \bar{\beta}}$ and $A_{\alpha \beta}=h_{\alpha \bar{\gamma}} A^{\bar{\gamma}}{ }_{\beta}$. We extend $\left(\omega_{\beta}{ }^{\alpha}\right)$ and $\tau^{\alpha}$ to forms on $U$ by

$$
\omega_{\beta}^{\alpha}(N)=0, \quad \text { and } \quad \tau^{\alpha}(N)=0
$$


Theorem 2.1 (C. R. Graham and J. M. Lee [5]). There exist uniquely determined 1-forms $\left(\varphi_{\beta}{ }^{\alpha}\right)$ such that

$$
\left.\varphi_{\beta}{ }^{\alpha}\right|_{M^{\varepsilon}}=\omega_{\beta}{ }^{\alpha}, \quad d h_{\alpha \bar{\beta}}=\varphi_{\alpha \bar{\beta}}+\varphi_{\bar{\beta} \alpha}
$$

and

(2.4) $d \theta^{\alpha}=\theta^{\beta} \wedge \varphi_{\beta}{ }^{\alpha}-i A^{\alpha}{ }_{\bar{\beta}} \theta^{0} \wedge \theta^{\bar{\beta}}+\frac{r}{2} \theta^{0} \wedge \theta^{\alpha}+\frac{r}{2} \theta^{\overline{0}} \wedge \theta^{\alpha}-\left(W^{\alpha} r\right) \theta^{0} \wedge \theta^{\overline{0}}$, where $W^{\alpha}=W_{\bar{\beta}} h^{\bar{\beta} \alpha}$.

The Graham-Lee ambient connection $\nabla$ is a complex linear connection defined by

$$
\nabla W_{\alpha}=\varphi_{\alpha}^{\beta} \otimes W_{\beta} \text { and } \nabla W_{0}=0 .
$$

We may assume that $\left\{W_{\alpha}\right\}$ was chosen that $h_{\alpha \bar{\beta}}=\delta_{\alpha \bar{\beta}}$. We call such $\{\xi=$ $\left.W_{0}, W_{1}, \ldots, W_{n}\right\}$ an adapted frame for $\varphi$. The curvature form of the GrahamLee ambient connection is $\Theta_{\beta}^{\alpha}=d \varphi_{\beta}^{\alpha}-\varphi_{\beta}^{\gamma} \wedge \varphi_{\gamma}^{\alpha}$.

Theorem 2.2 (C. R. Graham and J. M. Lee [5]). Let $\left\{\theta^{0}, \theta^{\alpha}\right\}$ be the coframe for an adapted frame. Then the curvature form of the Graham-Lee ambient curvature tensor is given by

$$
\begin{aligned}
\Theta_{\beta}{ }^{\alpha}= & \widetilde{R}_{\alpha}{ }^{\beta} \rho \bar{\gamma} \theta^{\rho} \wedge \theta^{\bar{\gamma}}+i \theta_{\alpha} \wedge \tau^{\beta}-i \tau_{\alpha} \wedge \theta^{\beta} \\
& +\left(i A_{\alpha \gamma, \bar{\beta}}-r_{\alpha} \delta_{\gamma}^{\beta}-\frac{1}{2} r_{\gamma} \delta_{\alpha}^{\beta}\right) \theta^{\gamma} \wedge \theta^{\overline{0}} \\
& -\left(i A_{\bar{\beta} \bar{\gamma}, \alpha}+r_{\bar{\beta}} \delta_{\bar{\gamma}}^{\bar{\alpha}}+\frac{1}{2} r_{\bar{\gamma}} \delta_{\alpha}^{\beta}\right) \theta^{0} \wedge \theta^{\bar{\gamma}} \\
& +\left(r_{\alpha} \delta_{\gamma}^{\beta}+\frac{1}{2} r_{\gamma} \delta_{\alpha}^{\beta}\right) \theta^{0} \wedge \theta^{\gamma}-\left(r_{\bar{\beta}} \delta_{\bar{\gamma}}^{\bar{\alpha}}+\frac{1}{2} r_{\bar{\gamma}} \delta_{\alpha}^{\beta}\right) \theta^{\overline{0}} \wedge \theta^{\bar{\gamma}} \\
& -\frac{1}{2}\left(r_{\alpha}{ }^{\beta}+r^{\beta}{ }_{\alpha}+2 A_{\alpha \gamma} A_{\bar{\gamma} \bar{\beta}}\right) \theta^{0} \wedge \theta^{\overline{0}},
\end{aligned}
$$

where $\widetilde{R}_{\alpha \rho \bar{\gamma}}^{\beta}$ is the pseudohermitian curvature tensor and $A_{\alpha \beta, \bar{\gamma}}$ is the coefficient of the pseudohermitian covariant derivative of $A$ on each $M^{\varepsilon}$.

Here and in the sequel, for a $k$-form $B=B_{i_{1} i_{2} \cdots i_{k}} \theta^{i_{1}} \wedge \theta^{i_{2}} \wedge \cdots \wedge \theta^{i_{k}}$, we denote the Graham-Lee covariant derivatives of the coefficients by adding indices after comma.

\section{Relations between the Graham-Lee ambient connection and the Levi-Civita connection}

Let $\Omega=\left\{z \in \mathbb{C}^{n+1}: \varphi(z)<0\right\}$ be a strongly pseudoconvex domain in $\mathbb{C}^{n+1}, n \geq 1$ with a defining function $\varphi$ such that $-\log (-\varphi)$ is strictly plurisubharmonic on a neighborhood of $\bar{\Omega}$. Let $g$ be a complete Kähler metric on $\Omega$ with the Kähler form

$$
\omega=-\partial \bar{\partial} \log (-\varphi)
$$


Let $\left\{W_{0}, W_{\alpha}\right\}$ be an adapted frame for $\varphi$ and $\left\{\theta^{0}, \theta^{\alpha}\right\}$ be its dual coframe. Let $\left(\psi_{k}{ }^{j}\right)$ be the connection 1-form of the Levi-Civita connection of $g$ which is uniquely determined by

$$
d \theta^{j}=\theta^{k} \wedge \psi_{k}^{j} \quad \text { and } \quad d g_{j \bar{k}}=\psi_{j \bar{k}}+\psi_{\bar{k} j},
$$

where $\psi_{j \bar{k}}=\psi_{j}^{l} g_{l \bar{k}}$.

Proposition 3.1 (cf. N. Seshadri [14], E. Barletta [1]). With respect to an adapted frame $\left\{W_{0}, W_{\alpha}\right\}$ for $\varphi, g$ is determined by equations

$$
g_{0 \overline{0}}=\frac{1-r \varphi}{\varphi^{2}}, \quad g_{0 \bar{\beta}}=0, \quad \text { and } \quad g_{\alpha \bar{\beta}}=-\frac{\delta_{\alpha \bar{\beta}}}{\varphi} .
$$

Moreover its Levi-Civita connection form $\left(\psi_{k}^{j}\right)$ is computed by the equations

$$
\begin{gathered}
\psi_{0}^{0}=-r \theta^{\overline{0}}+\left(r-\frac{1}{1-r \varphi}\left(\frac{2}{\varphi}-r+r_{0} \varphi\right)\right) \theta^{0}-\frac{r_{\alpha} \varphi}{1-r \varphi} \theta^{\alpha}, \\
\psi_{\alpha}^{0}=-\theta^{\bar{\alpha}}-\frac{r_{\alpha} \varphi}{1-r \varphi} \theta^{0}+i \frac{\varphi}{1-r \varphi} \tau^{\bar{\alpha}}, \\
\psi_{0}^{\alpha}=-i \tau^{\alpha}-r_{\bar{\alpha}} \theta^{\overline{0}}-\frac{1-r \varphi}{\varphi} \theta^{\alpha},
\end{gathered}
$$

and

$$
\psi_{\beta}{ }^{\alpha}=\varphi_{\beta}{ }^{\alpha}-\frac{r}{2} \delta_{\beta}^{\alpha} \theta^{\overline{0}}+\left(\frac{r}{2}-\frac{1}{\varphi}\right) \delta_{\beta}^{\alpha} \theta^{0} .
$$

Remark 3.2. By Proposition3.1, $\left\{e_{0}, \ldots, e_{n}\right\}$ where $e_{0}=\frac{\varphi}{\sqrt{1-r \varphi}} X_{0}$ and $e_{\alpha}=$ $\sqrt{-\varphi} X_{\alpha}$ form a unitary frame on $U$ with respect to the metric $g$.

\section{Asymptotic behavior of the holomorphic sectional curvature for}

$$
n>1
$$

Let $\Omega_{i}^{j}$ and $R_{i k \bar{l}}^{j}$ be the Kähler curvature form and the curvature coefficients of the metric $g$ with respect to the adapted frame $\left\{W_{0}, \ldots, W_{n}\right\}$ given by the equation (3.3), that is,

$$
\Omega_{i}^{j}=d \psi_{i}^{j}-\psi_{i}^{l} \wedge \psi_{l}^{j}=R_{i k l}^{j} \theta^{k} \wedge \theta^{\bar{l}} .
$$

Let $Q_{i k \bar{l}}^{j}$ be the Kähler curvature coefficients of $g$ with respect to the unitary frame $\left\{e_{0}, \ldots, e_{n}\right\}$ given in Remark 3.2. Similar to the Chern pseudoconformal curvature tensor, define

$$
\begin{aligned}
P_{\alpha \gamma \bar{\sigma}}^{\beta}:= & Q_{\alpha \gamma \bar{\sigma}}^{\beta}-\frac{1}{n+2}\left(Q_{\alpha}^{\beta} \delta_{\gamma \bar{\sigma}}+Q_{\gamma}^{\beta} \delta_{\alpha \bar{\sigma}}+\delta_{\alpha}{ }^{\beta} Q_{\gamma \bar{\sigma}}+\delta_{\gamma}{ }^{\beta} Q_{\alpha \bar{\sigma}}\right) \\
& +\frac{Q}{(n+1)(n+2)}\left(\delta_{\alpha}^{\beta} \delta_{\gamma \bar{\sigma}}+\delta_{\gamma}{ }^{\beta} \delta_{\alpha \bar{\sigma}}\right),
\end{aligned}
$$

where $Q_{\alpha}^{\beta}=Q_{\alpha j \bar{j}}^{\beta}$ is the Ricci tensor and $Q=Q_{j}^{j}$ is the scalar curvature. 
In [8], it is proved that the holomorphic sectional curvature of $g$ at a $p \in \Omega$ converges to a constant -2 as $p$ tends to the boundary. More generally, we can prove the following:

Proposition 4.1. Let $\Omega$ be a bounded strongly pseudoconvex domain in $\mathbb{C}^{n+1}$ with $C^{\infty}$-smooth boundary. Suppose that $n>1$. Then

$$
P_{\alpha \gamma \bar{\sigma}}^{\beta}=-\varphi C_{\alpha \gamma \bar{\sigma}}^{\beta}+O\left(\varphi^{2}\right)
$$

where $C_{\alpha \gamma \bar{\sigma}}^{\beta}$ is the Chern pseudoconformal curvature tensor of $\partial \Omega$ for every $C^{4}$-smooth defining function $\varphi$ of $\Omega$ such that $-\log (-\varphi)$ is strictly plurisubharmonic on a one-sided neighborhood $U$ of $\partial \Omega$. Hence, in particular, $\partial \Omega$ is spherical if and only if $P_{\alpha \gamma \bar{\sigma}}^{\beta}=O\left(\varphi^{2}\right)$.

Proof. By Theorem 2.2 and Proposition 3.1, one see that

$$
\begin{aligned}
R_{\alpha \gamma \bar{\sigma}}^{\beta} & =2 \Omega_{\alpha}^{\beta}\left(W_{\gamma}, W_{\bar{\sigma}}\right) \\
& =\widetilde{R}_{\alpha \gamma \bar{\sigma}}^{\beta}+\frac{1-r \varphi}{\varphi}\left(\delta_{\alpha}^{\beta} \delta_{\gamma \bar{\sigma}}+\delta_{\gamma}^{\beta} \delta_{\alpha \bar{\sigma}}\right)-\frac{\varphi}{1-r \varphi} A_{\alpha \gamma} A_{\bar{\sigma}}^{\beta} .
\end{aligned}
$$

Therefore,

$$
\begin{aligned}
Q_{\alpha \gamma \bar{\sigma}}^{\beta} & =-\varphi R_{\alpha \gamma \bar{\sigma}}^{\beta} \\
& =-(1-r \varphi)\left(\delta_{\alpha}^{\beta} \delta_{\gamma \bar{\sigma}}+\delta_{\gamma}^{\beta} \delta_{\alpha \bar{\sigma}}\right)-\varphi \widetilde{R}_{\alpha \gamma \bar{\sigma}}^{\beta}+\frac{\varphi^{2} A_{\bar{\sigma}}^{\beta} A_{\alpha \gamma}}{1-r \varphi} .
\end{aligned}
$$

Similarly, we also have

$$
\begin{aligned}
Q_{\alpha 0 \overline{0}}^{\beta} & =\frac{\varphi^{2}}{1-r \varphi} R_{\alpha 0 \overline{0}}^{\beta} \\
& =-\frac{\delta_{\alpha}^{\beta}}{1-r \varphi}+\frac{r \varphi}{1-r \varphi} \delta_{\alpha}^{\beta}-\frac{\varphi^{2}}{1-r \varphi} V_{\alpha}^{\beta} \\
& =-\delta_{\alpha}^{\beta}+O\left(\varphi^{2}\right),
\end{aligned}
$$

where

$$
V_{\alpha}^{\beta}=\left(\frac{r_{0}}{2}+\frac{r_{\overline{0}}}{2}+r^{2}\right) \delta_{\alpha}^{\beta}+\frac{r_{\alpha} r_{\bar{\beta}}}{1-r \varphi}+\frac{1}{2}\left(r_{\alpha}{ }^{\beta}+r_{\alpha}^{\beta}+2 \sum_{\gamma} A_{\alpha \gamma} A_{\bar{\gamma} \bar{\beta}}\right) .
$$

Contracting indices, we see that

$$
\begin{aligned}
Q_{\alpha}^{\beta} & =Q_{\alpha \gamma \bar{\gamma}}^{\beta}+Q_{\alpha 0 \overline{0}}^{\beta} \\
& =-(n+2) \delta_{\alpha}^{\beta}+\varphi\left((n+1) r \delta_{\alpha}^{\beta}-\widetilde{R}_{\alpha}^{\beta}\right)+O\left(\varphi^{2}\right) .
\end{aligned}
$$

Again, by Theorem 2.2 and Proposition 3.1

$$
\begin{gathered}
Q_{0 \alpha \bar{\alpha}}^{0}=-\varphi R_{0 \alpha \bar{\alpha}}^{0}=-1+O\left(\varphi^{2}\right), \\
Q_{0 \text { O } \overline{0}}^{0}=\frac{\varphi^{2}}{1-r \varphi} R_{00 \overline{0}}^{0}=-2+O\left(\varphi^{3}\right) .
\end{gathered}
$$


HIGHER ORDER ASYMPTOTIC BEHAVIOR OF COMPLETE KÄHLER METRICS 119

Therefore

$$
Q_{0}^{0}=Q_{0 \alpha \bar{\alpha}}^{0}+Q_{0}^{0} 0 \overline{0}=-2-n+O\left(\varphi^{2}\right) .
$$

Hence

$$
Q=Q_{\alpha}^{\alpha}+Q_{0}^{0}=-(n+2)(n+1)+\varphi(n(n+1) r-\widetilde{R})+O\left(\varphi^{2}\right) .
$$

By substituting (4.2), (4.4) and (4.7) in (4.1), we obtain

$$
\begin{aligned}
P_{\alpha \gamma \bar{\sigma}}^{\beta}= & -\varphi \widetilde{R}_{\alpha \gamma \bar{\sigma}}^{\beta}+\frac{\varphi}{n+2}\left(\widetilde{R}_{\alpha}^{\beta} \delta_{\gamma \bar{\sigma}}+\widetilde{R}_{\gamma}^{\beta} \delta_{\alpha \bar{\sigma}}+\delta_{\alpha}^{\beta} \widetilde{R}_{\gamma \bar{\sigma}}+\delta_{\gamma}{ }^{\beta} \widetilde{R}_{\alpha \bar{\sigma}}\right) \\
& -\frac{\varphi \widetilde{R}}{(n+1)(n+2)}\left(\delta_{\alpha}^{\beta} \delta_{\gamma \bar{\sigma}}+\delta_{\gamma}{ }^{\beta} \delta_{\alpha \bar{\sigma}}\right)+O\left(\varphi^{2}\right) \\
= & -\varphi C_{\alpha \gamma \bar{\sigma}}^{\beta}+O\left(\varphi^{2}\right),
\end{aligned}
$$

where $C_{\alpha \gamma \bar{\sigma}}^{\beta}$ is the Chern pseudoconformal curvature tensor of $\partial \Omega$. This implies the proposition.

Remark 4.2. Using similar method, for a nonvanishing section of $\mathcal{H}, Z$, one can find the expression

$$
H(Z)=-2-\varphi(\widetilde{H}(Z)-2 r)+O\left(\varphi^{2}\right)
$$

where $\widetilde{H}(Z)$ is the pseudohermitian sectional curvature in direction of $Z$ and

$$
H(\xi)=-2+O\left(\varphi^{3}\right) .
$$

Proof of Theorem 1.1(1). Since $H(Z)=-2+O\left(\varphi^{2}\right)$,

$$
Q_{\alpha \gamma \bar{\sigma}}^{\beta}=\frac{1}{2}\left(\delta_{\alpha}^{\beta} \delta_{\gamma \bar{\sigma}}+\delta_{\alpha \bar{\sigma}} \delta_{\gamma}^{\beta}\right)+O\left(\varphi^{2}\right) .
$$

By (4.3) and (4.10),

$$
Q_{\alpha}^{\beta}=\frac{n-1}{2} \delta_{\alpha}^{\beta}+O\left(\varphi^{2}\right)
$$

By (4.6) and (4.10),

$$
Q=\frac{1}{2}(n+1)(n-4)+O\left(\varphi^{2}\right) .
$$

Hence $P_{\alpha \gamma \bar{\sigma}}^{\beta}=O\left(\varphi^{2}\right)$ and therefore by Proposition 4.1, $\partial \Omega$ is spherical.

Corollary 4.3. Let $\Omega$ be a bounded strongly pseudoconvex domain with $C^{\infty}$ smooth boundary in $\mathbb{C}^{n+1}$. Let $g$ be the Bergman metric on $\Omega$ and $\rho$ be a $C^{\infty}$-smooth defining function of $\Omega$. If $n>1$ and

$$
H_{B}(W)=-\frac{4}{n+2}+O\left(\rho^{2}\right)
$$

for every nonzero complex vector $W$ where $H_{B}(W)$ denotes the holomorphic sectional curvature of the Bergman metric in direction $W$, then $\Omega$ is holomorphically covered by the unit ball in $\mathbb{C}^{n+1}$. 
Proof. Let

$$
\varphi(z)=-K(z, z)^{-1 /(n+2)},
$$

where $K$ is the Bergman kernel of $\Omega$. Then by Fefferman's asymptotic expansion formula for the Bergman kernel, $\varphi$ is of $C^{n+2, s}$-smooth defining function on $\bar{\Omega}$ for every $0<s<1$. By Theorem 1.1 (1), we get the conclusion.

\section{Asymptotic behavior of the holomorphic sectional curvature for}

$$
n=1
$$

Let us consider the case $n=1$. By Theorem 2.2, the curvature form of the Graham-Lee ambient connection for $n=1$ is given by

$$
\begin{aligned}
d \varphi_{1}{ }^{1}= & \widetilde{R} \theta^{1} \wedge \theta^{\overline{1}}+\left(i A_{11, \overline{1}}-\frac{3}{2} r_{1}\right) \theta^{1} \wedge \theta^{\overline{0}}-\left(i A_{\overline{1} \overline{1}, 1}+\frac{3}{2} r_{\overline{1}}\right) \theta^{0} \wedge \theta^{\overline{1}} \\
& +\frac{3}{2} r_{1} \theta^{0} \wedge \theta^{1}-\frac{3}{2} r_{\overline{1}} \theta^{\overline{0}} \wedge \theta^{\overline{1}}+\left(\Delta_{b} r-\left|A_{11}\right|^{2}\right) \theta^{0} \wedge \theta^{\overline{0}}
\end{aligned}
$$

where $\widetilde{R}$ is the pseudohermitian curvature and $\Delta_{b}$ is the sublaplacian of the pseudohermitian structure. The asymptotic behavior of the curvature tensor of the Levi-Civita connection with respect to the adapted frame is given by the following:

Proposition 5.1. With respect to an adapted frame $\left\{W_{0}, W_{1}\right\}$ for $\varphi$, denote $R_{i k \bar{l}}^{j}$ and $\widetilde{R}_{i k \bar{l}}^{j}$ be the curvature tensor of the Levi-Civita connection and the Graham-Lee ambient connection respectively. Then the followings hold:

$$
R_{1}^{1}{ }_{1 \overline{1}}=\frac{2}{\varphi}+\left(\widetilde{H}\left(W_{1}\right)-2 r\right)+O(\varphi)
$$

where $\widetilde{H}\left(W_{1}\right)$ is the pseudohermitian sectional curvature in direction of $W_{1}$,

$$
\begin{aligned}
& R_{10 \overline{0}}^{1}=R_{10 \overline{0}}^{1}=-\frac{1}{\varphi^{2}}+\frac{r}{\varphi}+\left(\Delta_{b} r-\left|A_{11}\right|^{2}-r^{2}-\frac{r_{0}+r_{\overline{0}}}{2}\right)+O(\varphi) \text {, } \\
& R_{10 \overline{1}}^{0}=R_{0}^{0}{ }_{1 \overline{1}}=\frac{1}{\varphi}+O(\varphi), \quad R_{1{ }_{1 \overline{1}}}^{0}=O(\varphi), \\
& R_{00 \overline{0}}^{0}=-\frac{2}{\varphi^{2}}+\frac{2 r}{\varphi}+\left(2\left|r_{1}\right|^{2}+W_{\overline{0}} r_{0}+3 r r_{0}+2 r r_{\overline{0}}+3 r^{3}\right) \varphi+O\left(\varphi^{2}\right) \\
& R_{0{ }_{0 \overline{1}}^{0}}^{0}=\overline{R_{0}^{0}{ }^{0} \overline{0}}=\overline{R_{10 \overline{0}}^{0}}=\left(W_{\overline{1}} r_{0}+2 r r_{\overline{1}}+2 i r_{1} A_{\overline{1} \overline{1}}\right) \varphi+O\left(\varphi^{2}\right), \\
& R_{0}^{1}{ }^{1} \overline{1}_{1}=-i\left(A_{\overline{1} \overline{1}, 0}+2 r A_{\overline{1} \overline{1}}\right)-i A_{\overline{1} \overline{1}}\left(r_{0}+r^{2}\right) \varphi+O\left(\varphi^{2}\right), \\
& R_{1{ }_{1 \overline{0}}}^{0}=i\left(A_{\overline{1} \overline{1}, 0}+2 r A_{\overline{1} \overline{1}}\right) \varphi+O\left(\varphi^{2}\right), \quad R_{0{ }_{0 \overline{0}}}^{1}=O\left(\varphi^{2}\right) .
\end{aligned}
$$


HIGHER ORDER ASYMPTOTIC BEHAVIOR OF COMPLETE KÄHLER METRICS 121

Proof. We verify only (5.3) and (5.4) in this proof. The other asymptotic formulas can be also obtained in the same way.

By (2.1),

$$
\begin{aligned}
R_{10 \overline{1}}^{1} & =2\left(d \psi_{1}{ }^{1}-\psi_{1}{ }^{l} \wedge \psi_{l}{ }^{1}\right)\left(W_{0}, W_{\overline{1}}\right) \\
& =2 d \varphi_{1}{ }^{1}\left(W_{0}, W_{\overline{1}}\right)-\frac{1}{2} r_{\overline{1}}-2 \psi_{1}^{l} \wedge \psi_{l}{ }^{1}\left(W_{0}, W_{\overline{1}}\right) .
\end{aligned}
$$

By (5.1) and

$$
\psi_{1}^{0}\left(W_{0}\right)=-\frac{r_{1} \varphi}{1-r \varphi}, \quad \psi_{0}^{1}\left(W_{0}\right)=0
$$

we get

$$
R_{10 \overline{1}}^{1}=-i A_{\overline{1} \overline{1}, 1}-2 r_{\overline{1}}-i \frac{r_{1} \varphi A_{\overline{1} \overline{1}}}{1-r \varphi}
$$

With this and

$$
\begin{aligned}
& R_{01 \overline{1}}{ }^{1}=g^{1 \overline{1}} R_{0 \overline{1} 1 \overline{1}}=g^{1 \overline{1}} R_{1 \overline{1} 0 \overline{1}}=R_{10 \overline{1}}{ }^{1}, \\
& R_{11 \overline{0}}{ }^{1}=g^{1 \overline{1}} R_{1 \overline{1} 1 \overline{0}}=\overline{g^{1 \overline{1}} R_{1 \overline{1} 0 \overline{1}}}=\overline{R_{0}{ }^{1}{ }^{1} \overline{1}},
\end{aligned}
$$

we get (5.3). Since

$$
\begin{aligned}
2 d \psi_{0}{ }^{1}\left(W_{0}, W_{\overline{1}}\right) & =2 d\left(-i A_{\overline{1} \overline{1}} \theta^{\overline{1}}-r_{\overline{1}} \theta^{\overline{0}}-\frac{1-r \varphi}{\varphi} \theta^{1}\right)\left(W_{0}, W_{\overline{1}}\right) \\
& =-i A_{\overline{1} \overline{1}, 0}-\frac{r}{2} i A_{\overline{1} \overline{1}}+\frac{1-r \varphi}{\varphi} i A_{\overline{1} \overline{1}}
\end{aligned}
$$

and

$$
\psi_{0}^{0}\left(W_{0}\right)=P, \psi_{0}{ }^{1}\left(W_{\overline{1}}\right)=-i A_{\overline{1} \overline{1}}, \psi_{0}{ }^{1}\left(W_{\overline{1}}\right)=-i A_{\overline{1} \overline{1}}, \psi_{1}^{1}\left(W_{0}\right)=\frac{r}{2}-\frac{1}{\varphi},
$$

where

$$
\begin{aligned}
P & =\left(r-\frac{1}{1-r \varphi}\left(\frac{2}{\varphi}-r+r_{0} \varphi\right)\right) \\
& =-\frac{2}{\varphi}-\left(r_{0}+r^{2}\right) \varphi+O\left(\varphi^{2}\right),
\end{aligned}
$$

we can get

$$
\begin{aligned}
R_{0}{ }^{1} \overline{1}_{\overline{1}} & =2\left(d \psi_{1}^{0}-\psi_{0}^{l} \wedge \psi_{l}{ }^{1}\right)\left(W_{0}, W_{\overline{1}}\right) \\
& =-i A_{\overline{1} \overline{1}, 0}-\frac{r}{2} i A_{\overline{1} \overline{1}}+\frac{1-r \varphi}{\varphi} i A_{\overline{1} \overline{1}}+i A_{\overline{1} \overline{1}} P-i A_{\overline{1} \overline{1}}\left(\frac{r}{2}-\frac{1}{\varphi}\right) \\
& =-i\left(A_{\overline{1} \overline{1}, 0}+2 r A_{\overline{1} \overline{1}}\right)-i A_{\overline{1} \overline{1}}\left(r_{0}+r^{2}\right) \varphi+O\left(\varphi^{2}\right) .
\end{aligned}
$$

In case $n=1$, the unique CR invariant $Q_{\overline{1} \overline{1}}$ is given as the following lemma in terms of the torsion coefficient $A_{\overline{1} \overline{1}}$ and pseudohermitian curvature $\widetilde{R}$. 
Lemma 5.2 (cf. $[3,18]$ ). Let $M$ be a strongly pseudoconvex $C R$ manifold of dimension 3 . Then the pseudoconformal invariant $Q_{\overline{1} \overline{1}}$ given in [3] is

$$
Q_{\overline{1} \overline{1}}=A_{\overline{1} \overline{1}, T}-\frac{2 i}{3} A_{\overline{1} \overline{1}, 1 \overline{1}}+\frac{i}{2} \widetilde{R} A_{\overline{1} \overline{1}}-\frac{1}{6} \widetilde{R}_{\overline{1} \overline{1}} .
$$

In particular, $M$ is spherical if and only if $Q_{\overline{1} \overline{1}}=0$ on $M$.

Although the formula in Lemma 5.2 is not given in $[3,18]$, we can get it by comparing the algebraic relation between the pseudohermitian structure equation in [18] and the pseudoconformal structure equation in [3], as Webster computed the pseudoconformal curvature tensor in terms of the pseudohermitian one in [18].

Now we are ready to prove (3) in Theorem 1.1:

Proposition 5.3. Suppose $n=1$ and $\varphi$ is at least $C^{5}$-smooth. If

$$
H(W)=-2+O\left(\varphi^{3}\right)
$$

for every nonvanishing section $W$ of $T^{1,0} \Omega$, then $\partial \Omega$ is spherical.

Proof. The assumption gives

$$
H\left(\frac{\xi}{\|\xi\|}+\mu \frac{Z}{\|Z\|}\right)=-2+O\left(\varphi^{3}\right)
$$

for every $Z \in \mathcal{H}$ and $\mu \in \mathbb{C}$. This implies that

So

$$
R_{1 \overline{1} 0 \overline{1}}=g_{1 \overline{1}}^{\frac{3}{2}} g_{0 \overline{0}}^{\frac{1}{2}} O\left(\varphi^{3}\right), \quad R_{0 \overline{1} 0 \overline{1}}=g_{1 \overline{1}} g_{0 \overline{0}} O\left(\varphi^{3}\right) \text {. }
$$

Then by (5.3),

$$
R_{10 \overline{1}}^{1}=O(\varphi), \quad R_{00 \overline{1}}^{1}=O(\varphi) .
$$

$$
-i A_{\overline{1} \overline{1}, 1}=2 r_{\overline{1}},
$$

and by (5.4),

$$
A_{\overline{1} \overline{1}, 0}+2 r A_{\overline{1} \overline{1}}=0
$$

on $M$. Differentiating (5.5), we have

$$
-i A_{\overline{1} \overline{1}, 1 \overline{1}}=2 r_{\overline{1} \overline{1}} .
$$

Since moreover, $\widetilde{R}=2 r$ on $M$, we have

$$
Q_{\overline{1} \overline{1}}=A_{\overline{1} \overline{1}, T}+r_{\overline{1} \overline{1}}+i r A_{\overline{1} \overline{1}} .
$$

On the other hand, differentiating (2.4) and extracting the coefficient of the term $\theta^{0} \wedge \theta^{\overline{0}} \wedge \theta^{\overline{1}}$, we have

$$
A_{\overline{1} \overline{1}, \overline{0}}+r A_{\overline{1} \overline{1}}+i r_{\overline{1} \overline{1}}=0 .
$$

Extracting (5.7) from (5.6), we see

$$
0=i A_{\overline{1} \overline{1}, T}-r A_{\overline{1} \overline{1}}+i r_{\overline{1} \overline{1}}=i Q_{\overline{1} \overline{1}}
$$

since $W_{\overline{0}}-W_{0}=i T$. This yields the conclusion. 
Corollary 5.4. Let $\Omega$ be a bounded strongly pseudoconvex domains with $C^{\infty}$ smooth boundary in $\mathbb{C}^{2}$ with $C^{\infty}$-smooth defining function $\rho$. Let $g$ be the Bergman metric on $\Omega$. If

$$
H_{B}(W)=-\frac{4}{3}+O\left(\rho^{3}\right)
$$

for every nonzero $W \in T \Omega$, then $\Omega$ is holomorphically covered by the unit ball in $\mathbb{C}^{2}$.

Proof. As in Corollary 4.3, let $K$ be the Bergman kernel on $\Omega$ and

$$
\varphi=-K^{-\frac{1}{3}}=-\frac{\rho}{\left(a+b \rho^{3} \log \rho\right)^{\frac{1}{2}}}
$$

for some $a, b \in C^{\infty}(\bar{\Omega}), a>0$ on $\partial \Omega$ by Fefferman's asymptotic expansion formula [4]. Then $\varphi \in C^{\infty}(\Omega) \cap C^{3, s}(\bar{\Omega})$. Although $\varphi$ is not $C^{5}$-smooth on $\bar{\Omega}$, we can still compute the CR invariant $Q_{\overline{1} \overline{1}}$ with $\varphi$, since the Levi form of $\varphi$ is infinitely differentiable in vector fields tangent to $\partial \Omega$ by (5.8). So by the Theorem $5.3, \Omega$ is covered by the unit ball.

Remark 5.5. (1) Note that (5.5) holds even in case $H(Z)=-2+O\left(\varphi^{2}\right)$. Differentiate (5.5) in direction of $W_{1}$ and take the real part. Since $\widetilde{R}=2 r$, we have

$$
\Delta_{b} \widetilde{R}-\operatorname{Im} A_{11, \overline{1} \overline{1}}=0
$$

on $M$. The left hand side of (5.9) is usually called the $C R Q$-curvature, which is a constant multiple of the coefficient of the log singularity of the Szegö kernel for the contact form $\theta$. See [6].

(2) The converse of Theorem 1.1 is also true in the sense that if a bounded strongly pseudoconvex domain $\Omega$ is covered by the unit ball, then there exists a defining function $\varphi$ satisfying the hypotheses of Theorem 1.1. In fact, since the Kähler-Einstein metric is preserved by holomorphic covering maps, we see that the Kähler-Einstein metric of any ball-quotient has constant negative holomorphic sectional curvature. On the other hand, it is well-known that the Bergman metric is not preserved by holomorphic covering maps. Qi-Keng $\mathrm{Lu}$ proved that the bounded domain which has the complete Bergman metric with constant holomorphic sectional curvature is biholomorphic to the unit ball (see [9]). In this point of view, one may ask that if the holomorphic sectional curvature of the Bergman metric of a bounded strongly pseudoconvex domain tends to a negative constant up to some order $k \geq 3$ (possibly, $k=\infty$ ), then in fact, the domain is biholomorphic to the unit ball. This seems to be an open question, which might depend on some global property of the Bergman metric.

\section{References}

[1] E. Barletta, On the boundary behavior of the holomorphic sectional curvature of the Bergman metric, Matematiche (Catania) 61 (2006), no. 2, 301-316. 
[2] S. S. Chern and S. Ji, On the Riemann mapping theorem, Ann. of Math (2) 144 (1996), no. 2, 421-439.

[3] S. S. Chern and J. K. Moser, Real hypersurfaces in complex manifolds, Acta Math. 133 (1974), 219-271.

[4] C. Fefferman, On the Bergman kernel and biholomorphic mappings of pseudoconvex domains, Bull. Amer. Math. Soc. 80 (1974), no. 4, 667-669.

[5] C. R. Graham and J. M. Lee, Smooth solutions of degenerate Laplacians on strictly pseudoconvex domains, Duke Math. J. 57 (1988), no. 3, 697-720.

[6] K. Hirachi, Scalar pseudo-Hermitian invariants and the Szegö kernel on three-dimensional CR manifolds, Complex geometry (Osaka, 1990), 67-76, Lecture Notes in Pure and Appl. Math., 143, Dekker, New York, 1993.

[7] K. T. Kim and J. Yu, Boundary behavior of the Bergman curvature in strictly pseudoconvex polyhedral domains, Pacific J. Math. 176 (1996), no. 1, 141-163.

[8] P. F. Klembeck, Kähler metrics of negative curvature, the Bergmann metric near the boundary, and the Kobayashi metric on smooth bounded strictly pseudoconvex sets, Indiana Univ. Math. J. 27 (1978), no. 2, 275-282.

[9] Q. K. Lu, On Kaehler manifolds with constant curvature, Acta Math. Sinica 16 (1966), 269-281 (Chinese); English transl. in Chinese Math. Acta 8 (1966), 283-298.

[10] N. Mok, The uniformization theorem for compact Kähler manifolds of nonnegative holomorphic bisectional curvature, J. Differential Geom. 27 (1988), no. 2, 179-214.

[11] S. Y. Nemirovskii and R. G. Shafikov, Uniformization of strictly pseudoconvex domains. I, Izv. Mat. 69 (2005), no. 6, 1189-1202.

[12] Uniformization of strictly pseudoconvex domains. II, Izv. Mat. 69 (2005), no. 6, 1203-1210.

[13] S. I. Pincuk, Proper holomorphic maps of strictly pseudoconvex domains, (Russian) Sibirsk. Mat. Z. 15 (1974), 909-917, 959.

[14] N. Seshadri, Volume renormalization for complete Einstein-Kähler metrics, Differential Geom. Appl. 25 (2007), no. 4, 356-379.

[15] Y.-T. Siu, The complex-analyticity of harmonic maps and the strong rigidity of compact Kähler manifolds, Ann. of Math. (2) 112 (1980), no. 1, 73-111.

[16] Y.-T. Siu and S.-T. Yau, Complete Kähler manifolds with nonpositive curvature of faster than quadratic decay, Ann. of Math. (2) 105 (1977), no. 2, 225-264.

[17] _ Compact Kähler manifolds of positive bisectional curvature, Invent. Math. 59 (1980), no. 2, 189-204.

[18] S. M. Webster, Pseudo-Hermitian structures on a real hypersurface, J. Differential Geom. 13 (1978), 25-41.

JAE-ChEON JOO

Department of MATHEMATICS AND INFORMATiCS

UNIVERSITY OF WUPPERTAL

Gaussstr. 20, D-421119 Wuppertal, Germany

E-mail address: jcjoo91@uni-wuppertal.de

AERYEONG SEO

SCHOOL OF MATHEMATICS

Korea Institute for Advanced Study (KIAS)

SEOUl 130-722, KoreA

E-mail address: Aileen83@kias.re.kr 